\title{
ESTABILIDADE DE PRODUÇÃO DE HÍBRIDOS SIMPLES E DUPLOS DE MILHO ORIUNDOS DE UM MESMO CONJUNTO GÊNICO $\left({ }^{1}\right)$
}

\author{
JUAREZ CAMPOLINA MACHADO $\left({ }^{2 *}\right)$; JOÃO CÂNDIDO DE SOUZA $\left({ }^{3}\right)$; \\ MAGNO ANTONIO PATTO RAMALHO $\left({ }^{3}\right)$; JOSÉ LUÍS LIMA $\left({ }^{3}\right)$
}

\begin{abstract}
RESUMO
O presente trabalho foi realizado com o objetivo de comparar a estabilidade de híbridos simples e híbridos duplos de milho oriundos de um mesmo conjunto gênico. Foram avaliados 55 tratamentos, sendo dez híbridos simples comerciais, utilizados como parentais e 45 híbridos duplos resultantes de um dialelo completo. As sementes dos híbridos duplos foram obtidas na área experimental do Departamento de Biologia da Universidade Federal de Lavras (DBI/UFLA). Os experimentos foram desenvolvidos em 15 ambientes, no ano agrícola de 2005/2006, em propriedades de agricultores e estações experimentais. O delineamento experimental utilizado foi o de blocos casualizados com três repetições e o caráter avaliado foi a produtividade de espigas despalhadas $\left(\mathrm{kg} \mathrm{ha}^{-1}\right)$, corrigida para $13 \%$ de umidade. Obteve-se a contribuição de cada híbrido para a interação genótipos $x$ ambientes e o desvio em relação ao desempenho máximo em cada ambiente utilizando a estatística não-paramétrica por meio da soma de postos. Os híbridos duplos foram, em média, mais estáveis, contudo, identificaram-se híbridos simples tão estáveis quanto os duplos.
\end{abstract}

Palavras-chave: Interação genótipos x ambientes, Estatística não-paramétrica, Zea mays L.

\section{ABSTRACT \\ YIELD STABILITY IN SINGLE AND DOUBLE CROSSES OF MAIZE ORIGINATED FROM THE SAME GENE POOL}

The objective of the present work was to study the adaptability and stability of single and doublecrosses of maize originated from the same gene pool. Ten commercial single-crosses and all possible double-crosses, obtained from a complete diallel, were evaluated. Seeds of the double-crosses were obtained in an experimental area of the Biology Department at Universidade Federal de Lavras (DBI/ UFLA). The experiments were conducted in 15 environments in the 2005/06 growing season, on farms and in experimental stations. The cultural practices were the ones normally used by farmers or experimental stations for maize. The entries were evaluated in randomized complete blocks design with three replications per environment. The trait under study was husked ears yield $\left(\mathrm{kg} \mathrm{ha}^{-1}\right)$, corrected to $13 \%$ of moisture content. Nonparametric statistics were used to study hybrids adaptability and stability. The contribution of each hybrid to the genotype-by-environment interaction and the deviation in relation to the maximum performance in each environment was determined. The double-crosses were on average more stable, although some single-crosses were as stable as the double-crosses.

Key words: Genotype by environment interaction, Nonparametric statistics, Zea mays L.

( $\left.{ }^{1}\right)$ Recebido para publicação em 14 de junho de 2007 e aceito em 10 de dezembro de 2007.

$\left({ }^{2}\right)$ Pós-Graduação em Genética e Melhoramento, Universidade Federal de Viçosa, 36571-000 Viçosa (MG). E-mail: juarezcmachado@yahoo.com.br $\left(^{*}\right)$ Autor correspondente. Bolsista do Conselho Nacional de Desenvolvimento Científico e Tecnológico (CNPq).

$\left({ }^{3}\right)$ Departamento de Biologia, Universidade Federal de Lavras (UFLA), Caixa Postal 3037, 37200-000 Lavras (MG). 


\section{INTRODUÇÃO}

O cultivo do milho no Brasil é realizado em uma ampla diversidade de ambientes. Existem, desde empresários rurais que cultivam extensas áreas e utilizam de todas as tecnologias disponíveis, como também os agricultores familiares que cultivam pequenas áreas e normalmente empregam baixa tecnologia. Nesse contexto, a interação genótipos $x$ ambientes é de fundamental importância, exigindo que os melhoristas utilizem todas as estratégias visando atenuar seus efeitos, e até mesmo aproveitála em benefício dos agricultores.

Entre as estratégias para manusear a interação, a escolha de cultivares é de fundamental importância. No Brasil, são vários os tipos de cultivares comercializadas, destacando os híbridos simples (HS), triplos (HT) e duplos (HD). O questionamento normalmente realizado, é se os híbridos duplos, devido à maior heterogeneidade genética, teriam maior estabilidade do que os HS (BECKER e LÉON, 1988; Guillen-Portal et al., 2003). Contudo há alguns relatos de HS que foram tão ou mais estáveis do que os HD (Ribeiro et al., 2000; Carvalho et al., 2005). Infelizmente, nesses trabalhos as comparações foram realizadas envolvendo diferentes combinações híbridas, em que os híbridos duplos não possuíam nenhuma relação com os híbridos simples. Seria importante que essas comparações fossem realizadas entre híbridos do mesmo conjunto gênico.

Por essa razão, foi realizado este trabalho visando comparar a estabilidade de híbridos simples e híbridos duplos obtidos dos respectivos híbridos simples.

\section{MATERIAL E MÉTODOS}

Foram utilizados dez híbridos simples comercias (A, B, C, D, E, F, G, H, I e J) de diferentes empresas (Monsanto, Pioneer, Nidera Sementes e Dow AgroScience), os quais diferiam quanto à textura dos grãos e ciclo. Esses HS foram escolhidos por serem recomendados para o cultivo na Região Sul de Minas Gerais. Empregando-se o esquema de cruzamento dialélico, foram obtidos todos os $45 \mathrm{HD}$ possíveis. As hibridações para obtenção dos HD foram realizadas na área experimental do Departamento de Biologia da Universidade Federal de Lavras (DBI/UFLA), no ano agrícola de 2004/ 2005.

Os experimentos foram desenvolvidos em 15 ambientes, no ano agrícola de 2005/06, em propriedades de agricultores e estações experimentais (Tabela 1). Os 55 tratamentos foram avaliados no delineamento de blocos casualizados com três repetições e a parcela experimental constituída de duas linhas de $3 \mathrm{~m}$ de comprimento, mantendo uma densidade populacional, após o desbaste, de 55.000 plantas por hectare. O caráter avaliado foi a produtividade de espigas despalhadas $\left(\mathrm{kg} \mathrm{ha}^{-1}\right)$, corrigida para $13 \%$ de umidade. Em cada ambiente, os tratos culturais foram os normalmente adotados pela instituição de pesquisa ou proprietário agrícola.

Procedeu-se a análise de variância por ambiente e posteriormente a análise conjunta, de acordo com os procedimentos preconizados por RAMALHO et al. (2005). A análise conjunta foi efetuada utilizando o ajuste do estande ideal pela covariância (Vencovsky e BARRIGA, 1992). Todas as análises foram realizadas no PROC GLM do SAS (SAS, 2000).

Avaliou-se a homogeneidade das variâncias residuais dos experimentos que, a princípio, foi verificada por meio do teste da razão entre o maior e o menor quadrado médio residual dos ensaios. Apesar da relativa homogeneidade observada - razão inferior a sete vezes, conforme GoMEs (1990), implementou-se também, o teste de Hartley (Ramalho et al., 2005). Diante da heterocedasticidade detectada, adotou-se a estatística não-paramétrica por meio da soma de postos para o estudo da adaptabilidade e da estabilidade dos híbridos em avaliação (TRUberg e HueHn, 2000; Machado, 2007).

A partir das médias dos híbridos, foi realizada a ordenação dos dados em uma tabela de dupla entrada entre híbridos (dispostos nas linhas) e ambientes (dispostos nas colunas), tendo o híbrido de menor média em cada ambiente recebido o posto $1 \mathrm{e}$ o de maior média, o posto 55. A partir dessa classificação, foi obtida a estimativa da ecovalência (Wricke e WEBER, 1986), utilizando-se a seguinte expressão:

$$
W_{i}{ }^{2}=\sum\left(R_{i j}-\bar{R}_{i .}-\bar{R}_{. j}+\bar{R}_{. .}\right)^{2}
$$

em que: $W_{i}$ : ecovalência; $R_{i j}$ : posto referente à observação do híbrido $i$ no ambiente $j ; \bar{R}_{i}$ : posto médio do híbrido $i$; $\bar{R}_{. j}$ : posto médio do ambiente $j$ e $\bar{R}$. corresponde à média geral.

Estimou-se também o desvio de cada híbrido em relação ao desempenho máximo em cada ambiente. Nesse caso, realizou-se a ordenação de todos os dados em uma tabela de dupla entrada entre híbridos (dispostos nas linhas) e ambientes (dispostos nas colunas), tendo o híbrido de menor média recebido o posto 1 e o de maior média, o posto 825. A partir dessa classificação, foi obtida a estimativa do parâmetro de adaptabilidade e estabilidade de Lin e BinNs (1988), utilizando-se a seguinte expressão: 


$$
P_{i}=\sum_{j=1}^{n}\left(R_{i j}-M_{j}\right)^{2} / 2 n
$$

em que: $P_{i}:$ índice de adaptabilidade e estabilidade do híbrido $i ; R_{i j}$ : posto referente à observação do híbrido $i$ no ambiente $j$; $M_{j}$ : posto máximo entre todos os híbridos no ambiente $j$ e $n$ é o número de ambientes.

As análises de adaptabilidade e estabilidade foram realizadas com o auxílio do aplicativo computacional R (R CoRe TEAm, 2006).

\section{RESULTADOS E DISCUSSÃO}

Primeiramente, vale salientar que embora ocorra dificuldade no manejo dos experimentos nas propriedades dos agricultores, torna-se necessário realizar trabalhos desta natureza, pois se espera que os resultados reflitam as condições para as quais as cultivares serão recomendadas (CECCARELLI et al., 2006). Dos 15 experimentos de avaliação, nove foram desenvolvidos em propriedades de agricultores, e os demais em instituições de pesquisa, com ampla variação nos tratos culturais. Apesar da diversidade de manejo, o modelo estatístico adotado explicou $87,6 \%$ da variação total, revelando a boa precisão experimental (Tabela 2). Devido a essa diversidade de ambientes, foi possível constatar, na análise conjunta, a significância da fonte de variação ambientes $(\mathrm{P} \leq 0,01)$ (Tabela 2). O mesmo ocorreu para fonte de variação híbridos $(\mathrm{P} \leq 0,01)$, permitindo inferir que há diferença entre os híbridos avaliados. Esse fato, associado com o comentado anteriormente para ambientes, possibilitou detectar diferença significativa $(P \leq 0,01)$ para a interação híbridos $x$ ambientes. Condição essa, indispensável, quando se pretende comparar a estabilidade de cultivares (Tabela 2).

Tabela 2. Resumo da análise de variância conjunta para produtividade de espigas despalhadas $\left(\mathrm{kg} \mathrm{ha}^{-1}\right)$ de dez híbridos simples (HS) e 45 híbridos duplos (HD) de milho, avaliados em 15 ambientes, no ano agrícola de 2005/2006

\begin{tabular}{lcc}
\hline Fonte de Variação & GL & QM \\
\hline Ambientes (A) & 14 & $794873096^{* *}$ \\
Repetição/ Ambiente & 29 & $6585709^{* *}$ \\
Híbridos (H) & 54 & $18097540^{* *}$ \\
HS & 9 & $19467194^{* *}$ \\
HD & 44 & $15885586^{* *}$ \\
HS vs HD & 1 & $103096622^{* *}$ \\
H x A & 756 & $2554880^{* *}$ \\
HS x A & 126 & $2928380^{* *}$ \\
HD x A & 616 & $2396571^{* *}$ \\
HS vs HD x A & 14 & $6158962^{* *}$ \\
Resíduo & 1566 & 1289652 \\
\hline CV (\%) & - & 13,9 \\
$R^{2}(\%)$ & - & 87,6 \\
DMS Tukey 5\% (kg ha $\left.{ }^{-1}\right)$ & - & 977,6 \\
Média (kg ha $\left.{ }^{-1}\right)$ & - & 8162 \\
Média HS (kg ha $\left.{ }^{-1}\right)$ & - & 8643 \\
Média HD (kg ha $\left.{ }^{-1}\right)$ & - & 8082 \\
\hline
\end{tabular}

** Significativo, pelo teste de F, a $1 \%$ de probabilidade.

Tabela 1. Características dos ambientes de condução dos experimentos

\begin{tabular}{|c|c|c|c|c|c|c|}
\hline Ambiente & Município & Latitude & Longitude & $\operatorname{DMS}\left({ }^{1}\right)$ & $\mathrm{CV}\left({ }^{2}\right)$ & $\begin{array}{c}\text { Produtividade } \\
\text { média }\end{array}$ \\
\hline & & & & $\mathrm{kg} \mathrm{ha}^{-1}$ & $\%$ & $\mathrm{~kg} \mathrm{ha}^{-1}$ \\
\hline Área Experimental DBI/UFLA & Lavras, MG & $21^{\circ} 13^{\prime} S$ & $44^{\circ} 58^{\prime} \mathrm{W}$ & 5193 & 14,1 & 10803 \\
\hline Área Experimental Geneze & Guarda-Mor, MG & $17^{\circ} 34^{\prime} S$ & $47^{\circ} 08^{\prime} \mathrm{W}$ & 2844 & 13,5 & 6212 \\
\hline Área Experimental Bionacional & Barreiras, BA & $12^{\circ} 08^{\prime} S$ & $45^{\circ} 00^{\prime} \mathrm{W}$ & 1851 & 12,0 & 4549 \\
\hline Área Experimental Prezzotto & Jussara, GO & $23^{\circ} 35^{\prime} S$ & $52^{\circ} 28^{\prime} \mathrm{W}$ & 2195 & 12,5 & 5152 \\
\hline Fazenda Vitorinha & Lavras, MG & $21^{\circ} 12^{\prime} S$ & $44^{\circ} 58^{\prime} \mathrm{W}$ & 4423 & 20,8 & 6246 \\
\hline Área Experimental Coopadap & São Gotardo, MG & $19^{\circ} 18^{\prime} \mathrm{S}$ & $46^{\circ} 03^{\prime} \mathrm{W}$ & 3107 & 11,3 & 8085 \\
\hline Fazenda Faepe & Ijaci, MG & $21^{\circ} 09^{\prime} \mathrm{S}$ & $44^{\circ} 56^{\prime} \mathrm{W}$ & 4551 & 10,1 & 13192 \\
\hline Fazenda Faepe & Ijaci, MG & $21^{\circ} 09^{\prime} \mathrm{S}$ & $44^{\circ} 56^{\prime} \mathrm{W}$ & 3248 & 10,7 & 8896 \\
\hline Fazenda Mato Dentro & Lavras, MG & $21^{\circ} 13^{\prime} S$ & $45^{\circ} 03^{\prime} \mathrm{W}$ & 4309 & 14,6 & 8685 \\
\hline Fazenda Morro do Guerra & Ribeirão Vermelho, MG & $21^{\circ} 10^{\prime} S$ & $45^{\circ} 04^{\prime} \mathrm{W}$ & 5092 & 17,1 & 8737 \\
\hline Fazenda Candeias & Candeias, MG & $20^{\circ} 46^{\prime} \mathrm{S}$ & $45^{\circ} 19^{\prime} \mathrm{W}$ & 3624 & 10,8 & 9876 \\
\hline Fazenda Chimarrão & Paracatu, MG & $17^{\circ} 13^{\prime} \mathrm{S}$ & $46^{\circ} 39^{\prime} \mathrm{W}$ & 3174 & 12,0 & 7768 \\
\hline Fazenda Milanez & Carrancas, MG & $21^{\circ} 24^{\prime} S$ & $44^{\circ} 38^{\prime} \mathrm{W}$ & 3189 & 12,8 & 7353 \\
\hline Fazenda dos Coelhos & Itutinga, MG & $21^{\circ} 23^{\prime} S$ & $44^{\circ} 46^{\prime} \mathrm{W}$ & 4691 & 17,2 & 8041 \\
\hline Fazenda da Pedra & Ingaí, MG & $21^{\circ} 22^{\prime} S$ & $44^{\circ} 45^{\prime} \mathrm{W}$ & 6304 & 16,0 & 9166 \\
\hline
\end{tabular}

$\left({ }^{1}\right)$ DMS = Diferença mínima significativa (Tukey 5\%); $\left({ }^{2}\right) \mathrm{CV}=$ Coeficiente de Variação. 
Verificou-se que a média dos HS diferiu da média dos HD. A superioridade média dos HS em relação aos HD foi de $6,9 \%$, considerando todos os ambientes. $\mathrm{O}$ híbrido simples mais produtivo (F) foi 3,3\% superior ao híbrido duplo de melhor desempenho (F x G) (Tabela 3), contudo, formaram um único grupo pelo teste de SсотT e KnOtT (1974). Esses resultados estão de acordo com a proposição de Wricke e Weber (1986). Segundo esses autores, se os valores genotípicos de híbridos simples seguem uma distribuição normal, a média dos diferentes tipos de híbridos obtidos a partir deles pode ser predita. Nessa condição, o híbrido simples mais produtivo deve ser $3 \%$ superior ao híbrido duplo de melhor desempenho, como constatado na tabela 3.

No Brasil, tem-se observado a substituição gradativa dos híbridos duplos pelos triplos e simples. As sementes dos híbridos simples e triplos são de custo mais elevado, o que pode inviabilizar sua adoção por grande contingente de agricultores (CRUZ e Pereira Filho, 2006, Sangor et al., 2006). Observouse que é possível selecionar híbridos duplos com produtividade média comparável aos melhores híbridos simples, os quais podem ser adotados pelos agricultores de menor poder aquisitivo, sem perda expressiva em produtividade.

No estudo da estabilidade, verificou-se, pelas estimativas de ecovalência $\left(\mathrm{W}_{\mathrm{i}}^{2} \%\right)$, que os híbridos duplos (HD) contribuíram, em média, com 1,73\% para a interação genótipos $\mathrm{x}$ ambientes, e os híbridos simples (HS), com $2,20 \%$, ou seja, em média, os HD contribuíram $21 \%$ menos para a interação que os HS (Tabela 3). Esses resultados permitem inferir que a maior heterogeneidade dos híbridos duplos proporciona maior tamponamento populacional (Allard e Bradshaw, 1964; Guillen-Portal et al., 2003).
Entretanto, podem ser identificados híbridos simples tão estáveis quanto os duplos (CARVALHO et al., 2005).

No presente trabalho, identificou-se híbridos simples com estimativas de $\mathrm{W}_{\mathrm{i}}{ }^{2} \%$ equivalentes às dos híbridos duplos, como por exemplo, o híbrido simples $\mathrm{F}$, que contribuiu com $0,55 \%$ para a interação, valor semelhante ao observado para os híbridos duplos mais estáveis (Tabela 3).

Considerando que o interesse é obter cultivares que se aproximem da produtividade máxima no maior número de ambientes, foi estimado o parâmetro $P_{i}$ (Tabela 4). No híbrido simples $F$ houve a menor estimativa de $\mathrm{P}_{\mathrm{i}}$, seguido do híbrido simples D e do híbrido duplo F x G. Essas cultivares foram as que mais se aproximaram das primeiras colocações nos diferentes ambientes, ou seja, possuem o menor desvio em relação ao máximo. No outro extremo estão os HD C x E, A x J e E x C, que mais se afastaram da produtividade máxima nos diferentes ambientes.

Em relação aos critérios, produtividade média de espigas despalhadas, contribuição para a interação genótipos $\mathrm{x}$ ambientes e a estimativa do desvio em relação ao máximo em cada ambiente, os híbridos que se destacaram foram o híbrido simples $\mathrm{F}$ e o híbrido duplo F x G, que associam alta produtividade média, baixa estimativa de $P_{\mathrm{i}}$ e estabilidade de produção nos ambientes avaliados (Tabelas 3 e 4). Infere-se que é possível recomendar cultivares que atendam às diferentes necessidades dos agricultores, ou seja, desde aqueles que empregam altos níveis de tecnologia exigindo alta uniformidade da lavoura, com a utilização de híbridos simples, como também atender os agricultores que empregam baixa tecnologia, utilizando-se híbridos duplos com menor custo de sementes, mas sem perda expressiva na produtividade.

Tabela 3. Produtividade média de espigas despalhadas (PED), acima da diagonal, em kg ha-1 e estimativas da ecovalência $\left(\mathrm{W}_{\mathrm{i}}^{2} \%\right)$, acima da diagonal inferior, utilizando a soma de postos, obtidas na avaliação de híbridos de milho, envolvendo 15 ambientes, no ano agrícola de 2005/2006

\begin{tabular}{lccccccccccc}
\hline Genitores & $\mathrm{A}$ & $\mathrm{B}$ & $\mathrm{C}$ & $\mathrm{D}$ & $\mathrm{E}$ & $\mathrm{F}$ & $\mathrm{G}$ & $\mathrm{H}$ & $\mathrm{I}$ & $\mathrm{J}$ & Média \\
\hline $\mathrm{A}$ & 2,24 & $8973 \mathrm{~b}$ & $8243 \mathrm{~d}$ & $6678 \mathrm{~g}$ & $7929 \mathrm{e}$ & $9036 \mathrm{~b}$ & $7958 \mathrm{e}$ & $8655 \mathrm{c}$ & $8264 \mathrm{~d}$ & $6635 \mathrm{~g}$ & $9013 \mathrm{~b}$ \\
$\mathrm{~B}$ & 1,56 & 2,54 & $8341 \mathrm{~d}$ & $8875 \mathrm{c}$ & $7971 \mathrm{e}$ & $8618 \mathrm{c}$ & $8738 \mathrm{c}$ & $8217 \mathrm{~d}$ & $8008 \mathrm{e}$ & $8597 \mathrm{c}$ & $9054 \mathrm{~b}$ \\
$\mathrm{C}$ & 1,27 & 1,83 & 2,33 & $7790 \mathrm{e}$ & $6531 \mathrm{~g}$ & $7907 \mathrm{e}$ & $8007 \mathrm{e}$ & $8242 \mathrm{~d}$ & $7783 \mathrm{e}$ & $8060 \mathrm{~d}$ & $7923 \mathrm{e}$ \\
$\mathrm{D}$ & 1,17 & 1,8 & 2,11 & 2,59 & $7971 \mathrm{e}$ & $8388 \mathrm{c}$ & $7163 \mathrm{f}$ & $7718 \mathrm{e}$ & $8497 \mathrm{c}$ & $7004 \mathrm{f}$ & $9187 \mathrm{~b}$ \\
$\mathrm{E}$ & 1,45 & 2,49 & 0,07 & 3,59 & 2,21 & $8397 \mathrm{c}$ & $8137 \mathrm{~d}$ & $7723 \mathrm{e}$ & $7956 \mathrm{e}$ & $8177 \mathrm{~d}$ & $7475 \mathrm{e}$ \\
$\mathrm{F}$ & 1,16 & 1,85 & 1,88 & 2,96 & 1,86 & 0,55 & $9344 \mathrm{a}$ & $8272 \mathrm{~d}$ & $8320 \mathrm{~d}$ & $8868 \mathrm{c}$ & $9656 \mathrm{a}$ \\
$\mathrm{G}$ & 1,45 & 1,58 & 2,41 & 0,29 & 2,74 & 0,32 & 1,92 & $7538 \mathrm{e}$ & $8019 \mathrm{e}$ & $8101 \mathrm{~d}$ & $8870 \mathrm{c}$ \\
$\mathrm{H}$ & 1,88 & 2,12 & 2,08 & 0,96 & 1,47 & 2,05 & 0,98 & 2,64 & $7885 \mathrm{e}$ & $8157 \mathrm{~d}$ & $8187 \mathrm{~d}$ \\
$\mathrm{I}$ & 2,06 & 1,63 & 1,99 & 2,25 & 2,28 & 1,68 & 1,25 & 2,14 & 3,00 & $8004 \mathrm{e}$ & $8225 \mathrm{~d}$ \\
$\mathrm{~J}$ & 0,36 & 2,91 & 2,46 & 0,75 & 1,41 & 1,02 & 3,49 & 1,58 & 1,36 & 2,30 & $8839 \mathrm{c}$ \\
\hline
\end{tabular}

Médias seguidas pela mesma letra pertencem ao mesmo grupo, pelo teste de ScotT e KNOTT (1974), a 5\% de probabilidade. 
Tabela 4. Estimativas do parâmetro $P_{i}$ utilizando a soma de postos, obtidas na avaliação de híbridos simples e duplos de milho, envolvendo 15 ambientes, no ano agrícola de 2005/2006

\begin{tabular}{lcccccccccc}
\hline Genitores & A & B & C & D & E & F & G & H & I \\
\hline A & 19539 & 13528 & 29072 & 80714 & 38932 & 14848 & 38877 & 24865 & 26147 & 84312 \\
B & - & 19982 & 30072 & 17848 & 41365 & 23748 & 20013 & 40917 & 39461 & 26672 \\
C & - & - & 40605 & 46095 & 93828 & 47787 & 41234 & 31469 & 46067 & 43484 \\
D & - & - & - & 10133 & 44192 & 30317 & 63821 & 47703 & 22229 & 72590 \\
E & - & - & - & - & 58442 & 30651 & 41148 & 48874 & 39108 & 31073 \\
F & - & - & - & - & - & 7874 & 11553 & 34819 & 29478 & 19040 \\
G & - & - & - & - & - & - & 19926 & 55476 & 38093 & 40311 \\
H & - & - & - & - & - & - & - & 38785 & 48121 & 38053 \\
I & - & - & - & - & - & - & - & - & 36079 & 44314 \\
J & - & - & - & - & - & - & - & - & - & 22521 \\
\hline
\end{tabular}

\section{CONCLUSÃO}

Os híbridos duplos foram, em média, mais estáveis, contudo, identificaram-se híbridos simples tão estáveis quanto os duplos.

\section{AGRADECIMENTOS}

Ao Conselho Nacional de Desenvolvimento Científico e Tecnológico (CNPq), pela bolsa concedida aos pós-graduandos envolvidos.

\section{REFERÊNCIAS}

ALLARD, R.W.; BRADSHAW, A.D. Implications of genotypeenvironment interactions in applied plant breeding. Crop Science, Madison, v. 4, n. 5, p. 503-508, 1964.

BECKER, H.C.; LÉON, J. Stability analysis in plant breeding. Plant Breeding, Berlin, v. 101, n. 1, p. 1-23, 1988.

CARVALHO, H.W.L. de; CARDOSO, M.J.; LEAL, M. de L. da S.; SANTOS, M.X. dos; TABOSA, J.N.; SOUZA, E.M. Adaptabilidade e estabilidade de cultivares de milho no Nordeste brasileiro. Pesquisa Agropecuária Brasileira, Brasília, v. 40, n. 5, p. 471-477, 2005.

CECCARELLI, S.; GRANDO, S.; BAUM, M. Plant breeding for dry areas. In: Anais do X Simpósio Sobre Atualização em Genética e Melhoramento de Plantas. Lavras: UFLA, 2006. p. 29-57.

CRUZ, J.C.; PEREIRA FILHO, I.A. Cultivares de milho disponíveis no mercado de sementes do Brasil para a safra 2006/07. 2006. Disponível em: <http://www.cnpms.embrapa.br/milho/ cultivares/index.php>. Acesso em: 31 jan. 2007.

GUILLEN-PORTAL, F.R.; RUSSELL, W.K.; BALTENSPERGER, D.D.; ESKRIDGE, K. M.; D'CROZ-MASON, N.E.; NELSON, L.A. Best types of maize hybrids for the western high plains of the USA. Crop Science, Madison, v. 43, n. 6, p. 2065-2070, 2003.

LIN, C.S.; BINNS M.R. A superiority measure of cultivar performance for cultivar $x$ location data. Canadian Journal of Plant Science, Ottawa, v. 68, n. 3, n. 1, p. 193-198, 1988.
MACHADO, J.C. Estabilidade de produção e da capacidade de combinação em híbridos de milho. 2007. 68f. Dissertação (Mestrado em Genética e Melhoramento de Plantas) Universidade Federal de Lavras, Lavras, MG.

GOMES, F.P. Curso de estatística experimental. 12. ed., Piracicaba: Nobel, 1990. 487 p.

R DEVELOPMENT CORE TEAM. R: A language and environment for statistical computing. Vienna, Áustria: $R$ Foundation for Statistical Computing, 2006.

RAMALHO, M.A.P.; FERREIRA, D.F.; OLIVEIRA, A.C. Experimentação em genética e melhoramento de plantas. 2.ed. Lavras: UFLA, 2005. 326p.

RIBEIRO, P.H.E.; RAMALHO, M.A.P.; FERREIRA, D.F. Adaptabilidade e estabilidade de genótipos de milho em diferentes condições ambientais. Pesquisa Agropecuária Brasileira, Brasília, v. 35, n. 11, p. 2213-2222, 2000.

SANGOI, L.; ERNANI, P.R.; SILVA, P.R.F. da; HORN, D.; SCHMITT, A.; SCHWEITZER, C.; MOTTER, F. Rendimento de grãos e margem bruta de cultivares de milho com variabilidade genética contrastante em diferentes sistemas de manejo. Ciência Rural, Santa Maria, v. 36, n. 3, p. 747-755, 2006.

\section{SASINSTITUTE. SAS/STAT User's Guide, Version 8. Cary, 2000.}

SCOTT, A.J.; KNOTT, M.A. A cluster analysis method for grouping means in the analysis of variance. Biometrics, Raleigh, v. 30, n. 3, p. 507-512, 1974.

TRUBERG, B.; HUEHN, M. Contributions to the Analysis of Genotype $\times$ Environment Interactions: Comparison of Different Parametric and Non-parametric Tests for Interactions with Emphasis on Crossover Interactions. Journal of Agronomy and Crop Science, Madison, v. 185, n. 4, p.267-274, 2000.

VENCOVSKY, R.; BARRIGA, P. Genética biométrica no fitomelhoramento. Ribeirão Preto: Revista Brasileira de Genética, 1992. 496p.

WRICKE, G.; WEBER, W.E. Quantitative genetics and selection in plant breeding. New York: W. de Gruyter, 1986. 406 p. 